\title{
Beating the Abbe Diffraction Limit in Confocal Microscopy via Nonclassical Photon Statistics
}

\author{
D. Gatto Monticone, ${ }^{1,2,3}$ K. Katamadze, ${ }^{4,5}$ P. Traina ${ }^{6}$ E. Moreva, ${ }^{6,7}$ J. Forneris ${ }^{1,2,3}$ I. Ruo-Berchera, ${ }^{6}$ P. Olivero, ${ }^{1,2,3}$ \\ I. P. Degiovanni, ${ }^{6}$ G. Brida, ${ }^{6}$ and M. Genovese ${ }^{3,6}$ \\ ${ }^{1}$ Physics Department and NIS Inter-departmental Centre-University of Torino, I-10125 Torino, Italy \\ ${ }^{2}$ Istituto Nazionale di Fisica Nucleare (INFN) Sezione Torino, I-10125 Torino, Italy \\ ${ }^{3}$ Consorzio Nazionale Interuniversitario per le Scienze Fisiche della Materia (CNISM) Sezione Torino, I-10125 Torino, Italy \\ ${ }^{4}$ M. V. Lomonosov Moscow State University, 119991 Moscow, Russia \\ ${ }^{5}$ Russian Academy of Sciences, Institute of Physics and Technology, 117218 Moscow, Russia \\ ${ }^{6}$ Istituto Nazionale di Ricerca Metrologica (INRiM), I-10135 Torino, Italy \\ ${ }^{7}$ International Laser Center of M. V. Lomonosov Moscow State University, 119991 Moscow, Russia
}

(Received 20 June 2014; published 30 September 2014)

\begin{abstract}
We experimentally demonstrate quantum enhanced resolution in confocal fluorescence microscopy exploiting the nonclassical photon statistics of single nitrogen-vacancy color centers in diamond. By developing a general model of superresolution based on the direct sampling of the $k$ th-order autocorrelation function of the photoluminescence signal, we show the possibility to resolve, in principle, arbitrarily close emitting centers.
\end{abstract}

DOI: 10.1103/PhysRevLett.113.143602

PACS numbers: 42.50.-p, 42.30.Va, 42.50.Ar, 42.50.St

In the last decade, measurement techniques enhanced by using peculiar properties of quantum light $[1,2]$ have been successfully demonstrated in several remarkable real application scenarios, for example, interferometric measurements aimed to reveal gravitational waves and the quantum gravity effect $[3,4]$, biological particle tracking [5], phase contrast microscopy [6], and imaging [7,8]. Very recently, a novel technique to beat the diffraction limit in microscopy that relies on the antibunching behavior of photons emitted by single fluophores has been proposed [9], and realized in wide field microscopy [10] by using an EMCCD camera.

The maximum obtainable imaging resolution in classical far-field fluorescence microscopy, according to the Abbe diffraction limit, is $R \simeq 0.61 \lambda / \mathrm{NA}$, where $\lambda$ is the wavelength of the light and NA is the numerical aperture of the objective. This restricts the current capability of precisely measuring the position of very small objects such as single photon emitters (color centers, quantum dots, etc.) [11-19], limiting their potential exploitation in the frame quantum technology [20,21]. In general, the research of methods to obtain a microscopy resolution below the diffraction limit is a topic of the utmost interest [22-29] that could provide dramatic improvement in the observation of several systems spanning from quantum dots [30] to living cells [31-34]. As a notable example, in several entanglement-related experiments using strongly coupled single photon emitters it is of the utmost importance to measure their positions with the highest spatial resolution [35]. In principle, this limitation can be overcome by recently developed microscopy techniques such as stimulated emission depletion (STED) and ground state depletion (GSD) [36,37]. Nevertheless, even if they have been demonstrated effectively able to provide superresolved imaging in many specific applications, among which are color centers in diamond [38], they are characterized by rather specific experimental requirements (dual laser excitation system, availability of luminescence quenching mechanisms by stimulated emission, nontrivial shaping of the quenching beam, high power). Furthermore, these techniques are not suitable in applications in which the fluorescence is not optically induced $[39,40]$, so that new methods are required for those applications.

Inspired by the works in [9], in this Letter we develop a comprehensive theory of superresolution imaging of clusters of single photon emitters based on high order Glauber correlation functions $g^{(k)}(t=0)$. Our theory discloses the unexpected possibility of approaching an arbitrary resolution just by measuring the spatial map of the correlation up to $k_{0}$ th order when it is reasonable to assume $g^{(k)}=0$ for $k>k_{0}$. For example, two arbitrarily closed emitters can be, in principle, separated just by measuring $g^{(2)}$ being of course $g^{(3)}=0$. Then, it confirms the indication of [9] that a fair $1 / \sqrt{k}$ improvement of resolution can be obtained with the measurement of $g^{(k)}$, if no further information is available. We experimentally test the theory of quantum superresolution in the significant case of confocal microscopy for the first time, considering clusters of few NV centers in artificial diamond grown by chemical vapor deposition and using a detector-tree of commercial (non-photon-number-resolving) single photon detectors $[18,41]$. We demonstrate a resolution increase by sampling the $g^{(2)}$ of the signal, and a further improvement by measuring $g^{(3)}$. Furthermore, we show that just by considering the contribution of higher powers of $g^{(2)}$, when only two centers are relevant (as certified by $g^{(3)}=0$ ), larger improvement in the resolution can be obtained, as predicted by the theory. This technique appears particularly valuable since the sampling of $g^{(2)}$ is a widely used and 
well-established experimental procedure to test the statistical properties of quantum optical sources in general, and of single photon sources in particular; thus, its adoption may come at almost zero cost.

Let $\mathcal{P}(x)$ be the probability of detecting a photon at the image position $x$ from a single photon emitter upon a pulsed excitation [42].

The function $\mathcal{P}(x)$ is typically an unimodal distribution and when fluorescence saturation effects are neglected the normalized $\mathcal{P}(x)$ represents the point spread function (PSF) of the microscope. In general, when taking the $k$ th power, $[\mathcal{P}(x)]^{k}$, the function gets narrower. In most cases $\mathcal{P}(x)$ can be well fitted by a Gaussian function, so the full width at half maximum (FWHM) of $[\mathcal{P}(x)]^{k}$ reduces by a factor $\sqrt{k}$.

The fluorescence signal $S$ coming from $n$ arbitrarily distributed emitters in a specific image position $x$ is then proportional to $S(x) \propto \sum_{\alpha=1}^{n} \mathcal{P}_{\alpha}(x)$. In order to obtain a resolution enhancement in the case of the $n$ single photon emitters, i.e., to resolve the presence and the position of two or more centers when they are closer than the PSF, it would be useful to have a function containing pure $k$ th powers of each single emitter probability $\sum_{\alpha=1}^{n}\left[\mathcal{P}_{\alpha}(x)\right]^{k}$. Unfortunately, the $k$ th power of the signal itself contains also the cross products terms (c.p.) multiplied by the appropriate multinomial coefficients, $S^{k}(x) \propto \sum_{\alpha=1}^{n}\left[\mathcal{P}_{\alpha}(x)\right]^{k}+$ c.p.

The method described in the following allows the removal of the contribution from the cross product terms using photon correlations, resulting in an effective increase of optical resolution.

To simplify our analysis and without any loss of generality, we consider that all the losses and inefficiencies are accounted for as part of the source of the quantum state; i.e., the state $\hat{\rho}_{\alpha}(x)$ corresponding to the single photon emission of the $\alpha$ th source that is detected at the position $x$ of the ideal (unit-quantum efficiency and photon-number resolving) single-photon detector can be expressed as

$$
\hat{\rho}_{\alpha}(x)=\mathcal{P}_{\alpha}(x)|1\rangle_{\alpha}\left\langle\left. 1\right|_{\alpha}+\left[1-\mathcal{P}_{\alpha}(x)\right] \mid 0\right\rangle_{\alpha}\left\langle\left. 0\right|_{\alpha} .\right.
$$

The multiphoton state generated by several $n$ singlephoton emitters is then $\hat{\rho}=\bigotimes_{\alpha=1}^{n} \hat{\rho}_{\alpha}$.

Defining the number of detected photons from the system of single-photon emitters $\hat{N}=\sum_{\alpha=1}^{n} \hat{a}_{\alpha}^{\dagger} \hat{a}_{\alpha}$, one obtains that $\langle\hat{N}\rangle=\operatorname{tr}[\hat{\rho} \hat{N}]=\sum_{\alpha=1}^{n} \mathcal{P}_{\alpha}(x)$.

In this case we define the $k$ th-order auto-correlation function as

$$
g^{(k)}=\frac{\left\langle\prod_{i=0}^{k-1}(\hat{N}-i)\right\rangle}{\langle\hat{N}\rangle^{k}} .
$$

[Note that in case of continuous wave excitation the functions have time dependence: we are dealing with the value of $g^{(k)}(t=0)$.] Knowing the value of $\langle\hat{N}\rangle$ and the set of $g^{(i)}(1 \leq i \leq k)$, an image with increased resolution can be ideally obtained at any order $k$. For instance, the expressions of the superresolved images for orders spanning from $k=2$ to $k=5$ are

$$
\begin{gathered}
\sum_{\alpha=1}^{2}\left[\mathcal{P}_{\alpha}(x)\right]^{2}=\langle\hat{N}\rangle^{2}\left[1-g^{(2)}\right] \\
\sum_{\alpha=1}^{3}\left[\mathcal{P}_{\alpha}(x)\right]^{3}=\langle\hat{N}\rangle^{3}\left[1-\frac{3}{2} g^{(2)}+\frac{1}{2} g^{(3)}\right] \\
\sum_{\alpha=1}^{4}\left[\mathcal{P}_{\alpha}(x)\right]^{4}=\langle\hat{N}\rangle^{4}\left\{1-2 g^{(2)}+\frac{1}{2}\left[g^{(2)}\right]^{2}+\frac{2}{3} g^{(3)}-\frac{1}{6} g^{(4)}\right\} \\
\sum_{\alpha=1}^{5}\left[\mathcal{P}_{\alpha}(x)\right]^{5}=\langle\hat{N}\rangle^{5}\left\{1-\frac{5}{2} g^{(2)}+\frac{5}{4}\left[g^{(2)}\right]^{2}+\frac{5}{6} g^{(3)}+\right. \\
\left.-\frac{5}{12} g^{(2)} g^{(3)}-\frac{5}{24} g^{(4)}+\frac{1}{24} g^{(5)}\right\}
\end{gathered}
$$

In general, the expressions of the superresolved images for any $k$ have the following form

$$
\sum_{\alpha=1}^{n}\left[\mathcal{P}_{\alpha}(x)\right]^{k}=\langle\hat{N}\rangle^{k} \sum_{i=1}^{i_{\max }} y_{i} \beta_{i}
$$

where $\beta_{i}$ represent in general products of the form $g^{\left(j_{1}\right)} g^{\left(j_{2}\right)} \ldots g^{\left(j_{l}\right)}, i_{\max }$ is the number of possible (ordered) combinations, satisfying the condition $\sum_{p=1}^{l} j_{p}=k$ [note that $g^{(1)}$ is equal to 1 according to Eq. (7)], and $y_{i}$ are multiplicative coefficients that can be straightforwardly calculated, as it is shown in the cases up to $k=5$ in Eqs. (3)-(6).

Figure 1 shows the setup used for our experiment, i.e., a laser scanning single photon sensitive confocal microscope. The excitation light emitted by a solid state laser at $532 \mathrm{~nm}$, coupled into a single mode fiber is collimated by a $4 \times$ objective. A dichroic mirror (long-pass at $570 \mathrm{~nm}$ ) reflects the excitation light ( $3 \mathrm{~mW}$ maximum) inside the

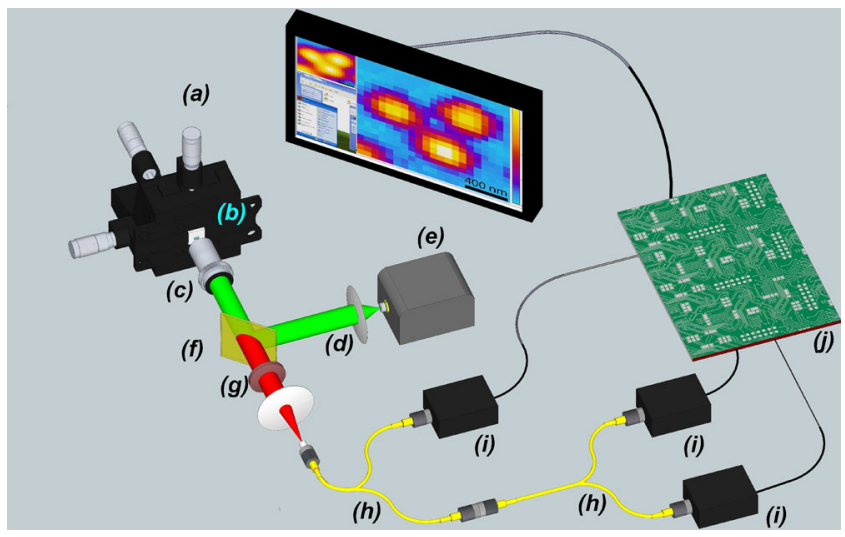

FIG. 1 (color online). Setup of the experiment: (a) XYZ closedloop piezoelectric stage, (b) sample, (c) $100 \times$ oil objective, (d) excitation light (532 nm), (e) laser source, (f) dichroic filter, (g) long-pass filters, (h) 50:50 fiber beam splitter, (i) singlephoton detectors, and (j) coincidence electronics. 
oil immersion objective (Olympus, $100 \times, \mathrm{NA}=1.3$ ) focusing inside the sample and transmits the fluorescence light towards the detecting apparatus. The sample (Element $\mathrm{Six}^{T M}$ electronic-grade polycrystalline chemical vapor deposition diamond) is mounted on a closed-loop $X Y Z$ piezoelectric stage, remotely controlled via PC, allowing nanometricresolution positioning in a $80 \mu \mathrm{m} \times 80 \mu \mathrm{m}$ area range. The fluorescence light (occurring within a $640-800 \mathrm{~nm}$ spectral window) is collected by the same objective used for excitation and then passes through the dichroic mirror and a long-pass filter in order to obtain a suitable attenuation $\left(>10^{12}\right)$ of the pump component. The signal is then focused by a $f=100 \mathrm{~mm}$ achromatic doublet and coupled to a $50 \mu \mathrm{m}$ multimode optical fiber that not only delivers the signal to the detectors, but also acts as a pinhole for the confocal system. The fiber leads to a detector-tree configuration $[18,41]$ realized by means of two integrated 50:50 beam splitters in cascade connecting to three single-photon avalanche photodiodes (Perkin-Elmer SPCM-AQR), operating in Geiger mode. This configuration, reproducing a generalized version of the "Hanbury Brown-Twiss" (HBT) interferometer [43], allows the detection of two- or threefold coincidences and the direct sampling of the values of the second order $\left(g^{(2)}\right)$ and third order $\left(g^{(3)}\right)$ [44-46] autocorrelation functions [47]. The signal counts and coincidences are measured via a picosecond time-tagging module (PicoQuant Hydra-Harp).

The first significant experimental results, demonstrating this method, are shown in Fig. 2. In the first section (a), a typical photoluminescence map of an area of the sample obtained via our confocal microscope is shown. One can observe that in some cases the centers are well separated, while in other cases they are too close to each other to be resolved by acquiring only the fluorescence signals. For instance, in the enlarged picture (b) a cluster of centers is shown that can be barely recognized as a unresolved group of three emitters. In (c),(d) the maps of the (respectively) $g^{(2)}$ and $g^{(3)}$ functions are shown. Here, the presence of three resolved $\mathrm{NV}$ centers is evident from the low value of $g^{(2)}$ and $g^{(3)}$ in correspondence of the center's positions, while in the surrounding region the values reach 1 because of background fluorescence light. Finally the superresolved maps for $k=2$ as in Eq. (3) and $k=3$ as in Eq. (4) are reported in the insets (e) and (f). The progressive increase of the resolution in the above-mentioned maps for increasing values of $k$ can be evaluated by comparing the width of the intensity PSF with the FWHMs of the superresolved maps. The intrinsic resolution of our microscope is estimated, by fitting the profiles of Fig. 2(b), to be $(500 \pm 16) \mathrm{nm}$, conservatively corresponding to the Abbe diffraction limit in the real experimental conditions. By fitting the profiles of Figs. 2(e) and 2(f), corresponding to $k=2$ and $k=3$, respectively, we obtained FWHM = $(360 \pm 30) \mathrm{nm}$ and FWHM $=(290 \pm 30) \mathrm{nm}$, confirming the expected resolution enhancement. Specifically, Fig. 3 shows the FWHM values compared with the expected
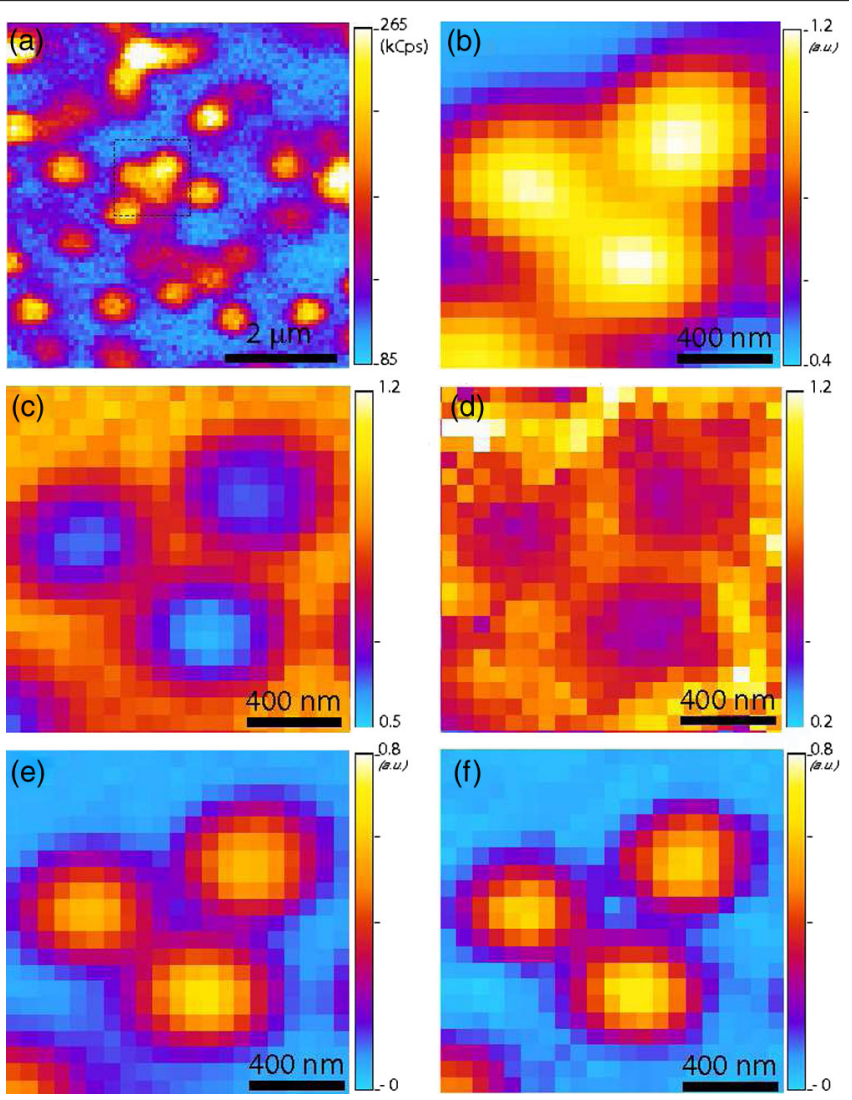

FIG. 2 (color online). Example of the super-resolution technique applied to a cluster of $3 \mathrm{NV}$ centers. (a) Typical scan on a region of the sample obtained collecting the signals emitted by each center on a pixel-by-pixel basis via single-photon sensitive confocal microscope. (b) Magnification of the area of interest. (c) Map of $g^{(2)}$ function. (d) Map of $g^{(3)}$ function. (e) Superresolved map for $k=2$. (f) Super-resolved map for $k=3$.

$1 / \sqrt{k}$ scaling of the resolution, where an excellent agreement is obtained, proving the substantial consistency of our data.

The advantages of our technique can be evinced even more from Fig. 4, where the photoluminescence signal from the observed region of the sample is mapped on a single-peaked spot (a) whose oval shape (with a major axis larger than $500 \mathrm{~nm}$ ) hints at the presence of more than one center, although no information on the quantity and relative position of these emitters can be extracted. Since the centers are very close to each other, even if the direct scanning of $g^{(2)}$ shown in (b) reveals the presence of two dips (i.e., two emitters), the superresolved map obtained for $k=2$ (c) is not able to separate them. No decisive improvement of the resolution of the image can be obtained by applying the third-order formula [see Eq. (4)], since only two emitters are present and the $g^{(3)}$ contribution is null everywhere, excluding background contribution that can be removed in the $g^{(2)}$ map [48]. Nonetheless, in this kind of scenario, a further improvement on the resolution can be achieved by applying the series of Eq. (7) at higher orders of $k$ with $g^{(k)}=0$ for $k \geq 3$. 


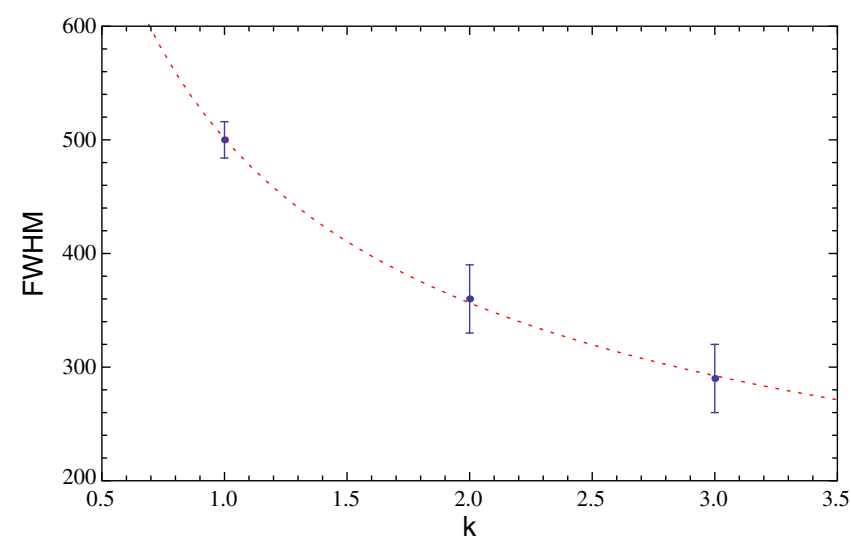

FIG. 3 (color online). Plot of the measured FWHM values, characterizing the resolution of the confocal microscope, versus the order $k$ of the generalized $g$ function used, together with the fit of the data, showing their good agreement with the expected scaling of the resolution as $1 / \sqrt{k}$.

As shown in the progression of (d),(e), and (f) of Fig. 4, the resolution increases at increasing $k$ orders (respectively, the third, fourth, and fifth) and eventually the positions of the two centers (or their distance) can be inferred with higher precision $(270 \pm 70 \mathrm{~nm})$.

In conclusion, we have experimentally demonstrated superresolved optical imaging of NV centers in bulk single-crystal artificial diamond by making smart use of the direct sampling of the $k$ th-order Glauber autocorrelation
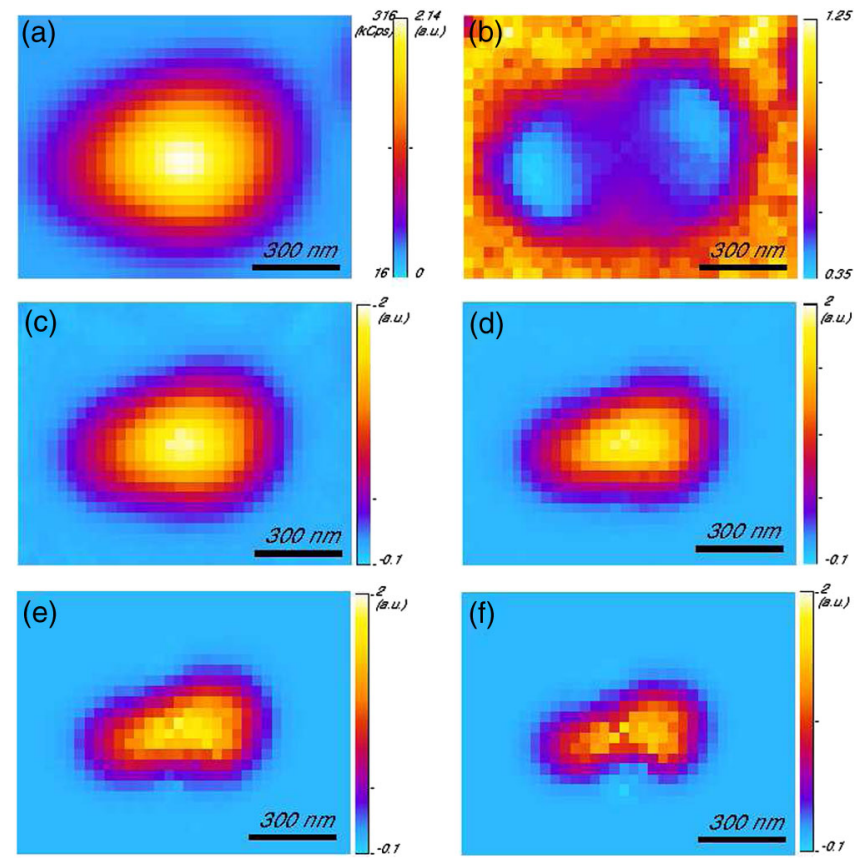

FIG. 4 (color online). (a) Direct mapping of the signal emitted by two NV centers, whose distance is below the FWHM of each peak, by the confocal microscope. (b) Map of the $g^{(2)}$ function. (c) Map obtained via the superresolution function for $k=2$. (d) Map obtained via the superresolution function for $k=3$. (e),(f) Maps derived, respectively, from Eq. (5) and Eq. (6) for $g^{(k)}=0$ for $k \geq 3$. function $g^{(k)}$ on a point-by-point basis (in particular, for $k=2,3)$. The analysis has been performed by feeding the signals acquired by our confocal microscope to single-photon detectors in a tree configuration. A comprehensive theory of the phenomenon enables two alternative scenarios. On one side, it predicts a narrowing of the PSF proportional to the square root of the highest order of the measured autocorrelation function. Moreover, if the further information that $g^{(k)}=0$ for $k>k_{0}$ is introduced in the model, arbitrary resolution can be reached in principle by using only up to the $k$ th order correlation. According to this, an experimental map distinguishing two very closed NV centers has been obtained, which would not be envisaged by the theory of Ref. [9], neither exploiting the intensity map nor by $g^{(2)}$ and even $g^{(3)}$ measurement. Therefore, the advantage of our technique has been demonstrated both theoretically and experimentally, paving the way for its use in several different experimental configurations, particularly in fields where the application of STED/GSD techniques are limited.

Furthermore, we note that the exploitation of the proposed technique is extremely straightforward to be implemented in microscopy systems investigating single-photon emitters, since they typically already make use of HBT interferometers for the $g^{(2)}$ measurements. Further enhancement of the resolution in the presence of multiple single photon emitters is achievable by measuring higher-order $g$ function, it is just necessary to increase the number of ports of the HBT interferometer, namely, the detector tree, a rather simple task due to the easy scalability of these detection systems [18]. Implementing a multiport HBT interferometer is useful not only for increasing the image resolution, but also because it has been proven to be a powerful diagnostic tool for quantum sources, not just single photon sources. Indeed, it was proven that by measuring $g^{(k)}$ with $k \geq 2$ one can reconstruct the mean number of photons of the different quantum optical modes of a quantum field [45]. For example, the optical modes of unwanted sources of background light superimposed to the emission of the single photon source of interest can be easily identified [45]. This can be of great interest for understanding the origin of this background and thus finding a way to eliminate it.

This research activity was supported by the following projects: FIRB Project No. D11J11000450001 funded by MIUR; EMRP Project No. EXL02-SIQUTE; "A.Di.NTech." Project No. D15E13000130003 funded by the University of Torino and Compagnia di San Paolo; NATO SPS Project No. 984397; "Compagnia di San Paolo" project "Beyond classical limits in measurements by means of quantum correlations."

[1] V. Giovannetti, S. Lloyd, and L. Maccone, Science 306, 1330 (2004).

[2] K. Banaszek, R. Demkowicz-Dobrzański, and I. A. Walmsley, Nat. Photonics 3, 673 (2009).

[3] J. Abadie et al., Nat. Phys. 7, 962 (2011). 
[4] I. Ruo Berchera, I. P. Degiovanni, S. Olivares, and M. Genovese, Phys. Rev. Lett. 110, 213601 (2013).

[5] M. A. Taylor, J. Janousek, V. Daria, J. Knittel, B. Hage, H.-A. Bachor, and W. P. Bowen, Nat. Photonics 7, 229 (2013).

[6] T. Ono, R. Okamoto, and S. Takeuchi, Nat. Commun. 4, 2426 (2013).

[7] G. Brida, M. Genovese, and I. Ruo Berchera, Nat. Photonics 4, 227 (2010).

[8] G. Brida, M. V. Chekhova, G. A. Fornaro, M. Genovese, E. D. Lopaeva, and I. Ruo Berchera, Phys. Rev. A 83, 063807 (2011).

[9] O. Schwartz and D. Oron, Phys. Rev. A 85, 033812 (2012).

[10] O. Schwartz, J. M. Levitt, R. Tenne, S. Itzhakov, Z. Deutsch, and D. Oron, Nano Lett. 13, 5832 (2013).

[11] C. Kurtsiefer, S. Mayer, P. Zarda, and H. Weinfurter, Phys. Rev. Lett. 85, 290 (2000).

[12] A. M. Zaitsev, Phys. Rev. B 61, 12909 (2000).

[13] D. Steinmetz, E. Neu, J. Meijer, W. Bolse, and C. Becher, Appl. Phys. B 102, 451 (2011).

[14] I. Aharonovich, C. Zhou, A. Stacey, J. Orwa, S. Castelletto, D. Simpson, A. Greentree, F. Treussart, J.-F. Roch, and S. Prawer, Phys. Rev. B 79, 235316 (2009).

[15] D. A. Simpson, E. Ampem-Lassen, B. C. Gibson, S. Trpkovski, F. M. Hossain, S. T. Huntington, A. D. Greentree, L. C. L. Hollenberg, and S. Prawer, Appl. Phys. Lett. 94, 203107 (2009).

[16] T. Müller, C. Hepp, B. Pingault, E. Neu, S. Gsell, M. Schreck, H. Sternschulte, D. Steinmüller-Nethl, C. Becher, and M. Atatüre, Nat. Commun. 5, 3328 (2014).

[17] D. Gatto Monticone et al., New J. Phys. 16, 053005 (2014).

[18] Single-Photon Generation and Detection, Experimental Methods in Physical Science Vol. 45, edited by A. Migdall, S. Polyakov, J. Fan, and J. Bienfang (Academic Press, New York, 2013), and references therein; https://www.elsevier .com/books/single-photon-generation-and-detection/migdall/ 978-0-12-387695-9.

[19] M. D. Eisaman, J. Fan, A. Migdall, and S. V. Polyakov, Rev. Sci. Instrum. 82, 071101 (2011).

[20] P. London, P. Balasubramanian, B. Naydenov, L. P. McGuinness, and F. Jelezko, Phys. Rev. A 90, 012302 (2014).

[21] J. Wrachtrup and F. Jelezko, J. Phys. Condens. Matter 18, S807 (2006).

[22] C. A. Pérez-Delgado, M. E. Pearce, and P. Kok, Phys. Rev. Lett. 109, 123601 (2012).

[23] C. Thiel, T. Bastin, J. Martin, E. Solano, J. von Zanthier, and G. Agarwal, Phys. Rev. Lett. 99, 133603 (2007).

[24] S. Oppel, T. Buttner, P. Kok, and J. vonZanthier, Phys. Rev. Lett. 109, 233603 (2012).

[25] V. N. Beskrovnyy and M. I. Kolobov, Phys. Rev. A 71, 043802 (2005).

[26] J. P. Dowling, Contemp. Phys. 49, 125 (2008).

[27] M. D'Angelo, M. V. Chekhova, and Y. Shih, Phys. Rev. Lett. 87, 013602 (2001).

[28] E. Distante, M. Jezek, and U. L. Andersen, Phys. Rev. Lett. 111, 033603 (2013).

[29] G. Björk, L. L. Sánchez-Soto, and J. Söderholm, Phys. Rev. A 64, 013811 (2001).

[30] O. A. Shcherbina, G. A. Shcherbina, M. Manceau, S. Vezzoli, L. Carbone, M. De Vittorio, A. Bramati, E.
Giacobino, M. V. Chekhova, and G. Leuchs, Opt. Lett. 39, 1791 (2014).

[31] R. Igarashi et al., Nano Lett. 12, 5726 (2012).

[32] D. Le Sage, K. Arai, D. R. Glenn, S. J. DeVience, L. M. Pham, L. Rahn-Lee, M. D. Lukin, A. Yacoby, A. Komeili, and R. L. Walsworth, Nature (London) 496, 486 (2013).

[33] S. Steinert, F. Ziem, L. T. Hall, A. Zappe, M. Schweikert, N. Götz, A. Aird, G. Balasubramanian, L. Hollenberg, and J. Wrachtrup, Nat. Commun. 4, 1607 (2013).

[34] M. A. Taylor, J. Janousek, V. Daria, J. Knittel, B. Hage, H.-A. Bachor, and W. P. Bowen, Phys. Rev. X4, 011017 (2014).

[35] F. Dolde, I. Jakobi, B. Naydenov, N. Zhao, S. Pezzagna, C. Trautmann, J. Meijer, P. Neumann, F. Jelezko, and J. Wrachtrup, Nat. Phys. 9, 139 (2013).

[36] S. W. Hell and J. Wichmann, J. Opt. Lett. 19, 780 (1994).

[37] S. W. Hell and M. Kroug, Appl. Phys. B 60, 495 (1995).

[38] E. Rittweger, K. Young Han, S. E. Irvine, C. Eggeling, and S. W. Hell, Nat. Photonics 3, 144 (2009).

[39] N. Mizuochi et al., Nat. Photonics 6, 299 (2012).

[40] A. Lohrmann, S. Pezzagna, I. Dobrinets, P. Spinicelli, V. Jacques, J.-F. Roch, J. Meijer, and A. M. Zaitsev, Appl. Phys. Lett. 99, 251106 (2011).

[41] H. Paul, P. Torma, T. Kiss, and I. Jex, Phys. Rev. Lett. 76, 2464 (1996); L. A. Jiang, E. A. Dauler, and J. T. Chang, Phys. Rev. A 75, 062325 (2007); V. Schettini, S. Polyakov, I. P. Degiovanni, G. Brida, S. Castelletto, and A. Migdall, IEEE J. Quantum Electron. Sel. Topics 13978 (2007); A. Divochiy, F. Marsili, D. Bitauld, A. Gaggero, R. Leoni, F. Mattioli, A. Korneev, V. Seleznev, N. Kaurova, O. Minaeva, G. Gol'tsman, K. G. Lagoudakis, M. Benkhaoul, F. Lévy, and A. Fiore, Nat. Photonics 2, 302 (2008); E. A. Dauler, A. J. Kerman, B. S. Robinson, J. K. W. Yang, B. Voronov, G. Goltsman, S. A. Hamilton, and K. K. Berggren, J. Mod. Opt. 56, 364 (2009); D. Rosenberg, A. J. Kerman, R. J. Molnar, and E. A. Dauler, Opt. Express 21, 1440 (2013); K. Banaszek and I. A. Walmsley, Opt. Lett. 28, 52 (2003); M. J. Fitch, B. C. Jacobs, T. B. Pittman, and J. D. Franson, Phys. Rev. A 68, 043814 (2003); D. Achilles, C. Silberhorn, C. Sliwa, K. Banaszek, and I. A. Walmsley, Opt. Lett. 28, 2387 (2003).

[42] The whole treatment holds also in continuous wave excitation, considering $d \mathcal{P}(x)$ as the infinitesimal probability of detecting the photon within an infinitesimal time interval $d t$ (In this case the relation will be $d \mathcal{P}=I d t$, where $I$ is the photon emission intensity, measured as detected photons/s).

[43] R. Hanbury-Brown and R. Q. Twiss, Nature (London) 177, 27 (1956).

[44] M. Avenhaus, K. Laiho, M. V. Chekhova, and C. Silberhorn, Phys. Rev. Lett. 104, 063602 (2010).

[45] E. A. Goldschmidt, F. Piacentini, I. Ruo Berchera, S. V. Polyakov, S. Peters, S. Kück, G. Brida, I. P. Degiovanni, A. Migdall, and M. Genovese, Phys. Rev. A 88, 013822 (2013).

[46] M. J. Stevens, S. Glancy, S. W. Nam, and R. P. Mirin, Opt. Express 22, 3244 (2014).

[47] Note that the lack of balance of the splitting ratio among the different ports of the HBT is not a problem in this case since the (higher order) correlation functions $g^{(k)}$ are independent on the "effective" quantum efficiency [18].

[48] R. Brouri, A. Beveratos, J.-P. Poizat, and P. Grangier, Opt. Lett. 25, 1294 (2000). 